\title{
SELEÇÃO RECORRENTE FENOTÍPICA VISANDO À OBTENÇÃO DE UMA VARIEDADE SINTÉTICA DE MILHO (ZEA MAYS L.).
}

Lenon Romano Modesto, Elivelton Alves Lustri, Fernando José Vasconcelos Lemes Soares, Ronis Pereira Fernandes da Silva, Antonio Fluminhan Junior

Universidade do Oeste Paulista - UNOESTE. Curso de Agronomia, Presidente Prudente - SP. E-mail: fluminhan@unoeste.br

\section{RESUMO}

O milho (Zea mays L.) é uma das principais espécies agrícolas cultivadas no mundo, sendo que a sua produção pode ser encontrada em regiões com condições de solo e clima muito contrastantes. Este fato é determinante para que exista um estímulo para estabelecimento de um programa de melhoramento genético. A presente pesquisa teve como objetivos a produção de uma variedade sintética de milho a partir do intercruzamento de cultivares comerciais disponíveis no mercado, bem como a avaliação do seu desempenho agronômico, observado durante o processo de seleção recorrente fenotípica. Foi observado que a população selecionada apresenta uma grande homogeneidade, visando a criação de uma população uniforme exigida para o registro da cultivar. A avaliação do desempenho agronômico dos genótipos contribuiu para a geração de conhecimento básico necessário ao processo desenvolvimento desta nova variedade.

Palavras-chave: milho, variedade sintética, melhoramento genético, seleção recorrente fenotípica, Zea mays L.

\section{PHENOTYPIC RECURRENT SELECTION AIMING TO THE OBTENTON OF A SYNTHETIC VARIETY OF MAIZE (ZEA MAYS L.)}

\begin{abstract}
Corn (Zea mays) is a major crop species grown in the world, and its production can be found in areas with soil conditions and very contrasting weather. This fact is crucial for the establishment of genetic breeding programs. The present research aimed to produce a synthetic variety of maize from the interbreeding of cultivars available in the market, as well as to evaluate the agronomic performance observed during the phenotypic recurrent selection procedure. It was observed that the selected population showed a great homogeneity, creating a uniform population, which is required for the cultivar registration. The evaluation of the agronomic performance of genotypes contributed to the generation of basic knowledge required to process development of this new variety.
\end{abstract}

Keywords: maize; synthetic variety; genetic breeding; phenotypic recurrent selection; Zea mays L. 


\section{INTRODUÇÃO}

O milho (Zea mays L.) é uma espécie que, em função de seu potencial produtivo, composição química e valor nutritivo, constitui-se em um dos mais importantes cereais cultivados do mundo. Devido à sua multiplicidade de aplicações, quer na alimentação humana quer na alimentação animal, assume relevante papel sócioeconômico, além de constituir-se em indispensável matéria-prima impulsionadora de diversificados complexos agroindustriais (DOURADO NETO; FANCELLI, 2000).

As safras brasileiras de milho têm oscilado drasticamente nas últimas duas décadas, alternando anos em que a produção global superou a demanda interna com outros em que o montante produzido foi insuficiente para atender às necessidades do país (MUNDSTOCK; SILVA, 2005). A partir do ano 2000, as frequentes frustrações das safras de verão e da safrinha, o aumento da demanda pelo cereal e a redução da área cultivada têm causado um delicado quadro de abastecimento com este grão no país (HORN, 2004).

Graças à variabilidade natural da espécie e ao crescente conhecimento das plantas utilizadas, o ser humano passou a influir decisivamente na modificação dos vegetais utilizados, domesticando-os e promovendo contínuas transformações e o seu melhoramento genético (PATERNIANI, 1995). Segundo o autor, estes procedimentos permitiram a seleção de cultivares de milho adaptados às diversas condições de clima e solo no planeta.

Segundo Malacrida et al. (2002), para a escolha das sementes do milho, muitos agricultores deparam-se com um problema inicial, que é a grande quantidade de cultivares e híbridos existentes no mercado, sendo que a decisão final é sempre influenciada pela sugestão de representantes comerciais e vendedores, levando muitas vezes a resultados insatisfatórios ou, no mínimo, não conseguindo a máxima lucratividade com o seu trabalho.

Sabe-se que os melhores resultados de rendimento agrícola são obtidos quando os cultivares são desenvolvidos especialmente para adaptação às condições de clima e solo do local para onde ele for direcionado (BARBOSA, 1995).

A presente pesquisa teve como objetivos a produção de uma variedade sintética de milho a partir do intercruzamento de cultivares comerciais disponíveis no mercado, bem como a avaliação do seu desempenho agronômico, observado durante o processo de seleção recorrente fenotípica. 


\section{METODOLOGIA}

O presente trabalho foi conduzido na área experimental agrícola da Universidade do Oeste Paulista - UNOESTE, localizada no Campus II, na Rodovia Raposo Tavares, km 572, no município de Presidente Prudente, São Paulo, onde há predominância de solo característico Argissolo (Podzólico) e com topografia plana, com sistema de irrigação por aspersão, implantado na área adjacente à Horta.

Os materiais empregados na presente pesquisa, que proporcionaram 0 intercruzamento original para constituir a variedade sintética foram os híbridos: AG1051, AG8088, PZ242, PZ6718, SHS4050, SHS4090 e as cultivares locais: CRIOULA, e SELEGRÃOS 52. A geração pioneira foi desenvolvida no período de maio a outubro de 2013, a partir da mistura de quantidades iguais de sementes de cada uma das cultivares originais.

A área do plantio foi correspondente a 16 linhas de 60 metros, totalizando cerca de $900 \mathrm{~m}^{2}$ e, aproximadamente, 4.500 plantas. A densidade de plantio empregada foi de cerca de 6 sementes por metro linear no espaçamento de $0,20 \mathrm{~m} \times 0,90 \mathrm{~m}$. 0 desenvolvimento das plantas foi considerado normal, sendo a colheita realizada em meados de Novembro de 2013, seguidas por posterior plantio da segunda geração.
O plantio das sementes da população

Sint-2 foi realizado no ano agrícola 20132014, a partir de sementes oriundas da primeira geração de seleção. Nesta geração, o plantio foi realizado em 24 linhas de 70 metros, com implemento para plantio direto regulado para depositar 6 sementes por metro linear.

A seleção de espigas colhidas de plantas da geração Sint-1 foi realizada escolhendo-se aquelas que exibiam maior comprimento, maior número de fileiras de grãos, maior precocidade e tipo de grãos, em especial, o meio-dente. A classificação das sementes foi realizada com peneiras de dimensões variáveis, sendo que as selecionadas foram as de número 22 ou maiores. Esta população de sementes, denominada "Elite", foi utilizada para a produção da geração subsequente (Sint-2).

As sementes selecionadas foram utilizadas no plantio visando a obtenção de um material gradativamente mais homogêneo e superior em relação às diversas características. As seleções foram realizadas nas seguintes fases:

I - Pleno desenvolvimento vegetativo, para seleção das melhores plantas, com desbastes de plantas inferiores;

II - Véspera do florescimento feminino, para seleção de plantas polinizadoras com desbaste de plantas com espiguetas mal formadas; 
III - Na formação das espigas (após a polinização), para seleção das plantas com melhor formação de espigas (precocidade).

IV - Na colheita, para seleção das espigas com melhor formação de grãos.

V - Após a colheita, seleção das melhores sementes, verificando se há uniformidade de fileiras de grãos e os aspectos gerais de sanidade das sementes.

O experimento foi conduzido em delineamento experimental inteiramente casualizados (DIC), sendo que as avaliações foram realizadas através da análise de dados de 4 linhas de 5 metros amostradas ao acaso dentro da parcela experimental, cada linha contou com 19 plantas nas quais foram coletados dados levando em conta os seguintes parâmetros: altura de planta, altura de inserção da espiga, comprimento de espiga, número de fileiras de grãos por espiga, peso de espiga, peso dos grãos/planta e peso de grãos separados por 3 diferentes peneiras (tamanhos 18, 20 e 22).

A variável 'produção de grãos em diferentes peneiras' foi avaliada pela primeira vez nessa geração, visando uma posterior comparação com gerações futuras, procurando acompanhar as variações apresentadas nas sucessivas gerações da variedade em produção.

As análises estatísticas foram realizadas através do programa estatístico ASSISTAT $^{\circledast}$ (com referências em SILVA, 1996;
SILVA; AZEVEDO, 2002; 2006; 2009). Os resultados também foram submetidos à comparação de médias pelo teste de Tukey a $5 \%$ de probabilidade por meio do mesmo programa estatístico, para a comparação de contrastes entre médias dos indivíduos. Como medida de dispersão dos dados, foram calculados os coeficientes de variação (C.V.\%) para cada característica avaliada.

\section{RESULTADOS}

As análises de variância e respectivos testes $F$ mostraram que as parcelas amostradas tiveram resultados significativos para as variáveis altura de planta, a $5 \%$ de probabilidade, altura de inserção de espira e peso de espiga, a $1 \%$ de probabilidade (Tabela 01). Provavelmente, este resultado ocorreu pela variação ambiental comumente presente em experimentos conduzidos a campo, os quais não conseguem proporcionar de forma exatamente iguais durante o ciclo de produção diversos fatores como: água da irrigação, controle de pragas e plantas daninhas, características pedológicas, interceptação da radiação solar, entre outros fatores.

$\mathrm{Na}$ análise referente às demais variáveis: comprimento de espiga, número de fileiras de grãos nas espigas e, é possível afirmar a clara homogeneidade que a variedade conseguiu obter na sua segunda geração de seleção. Os resultados obtidos 
com o teste $\mathrm{F}$ demonstram claramente que não foram encontradas diferenças significativas entre as parcelas. Os coeficientes de variação (C.V.) apresentados são em sua maioria baixos o que comprova a homogeneidade da variedade, com apenas algumas variáveis com dispersão do mesmo que serão trabalhadas para melhora nas próximas gerações.

Tabela 01. Valores de $\mathrm{F}$ e coeficientes de variação (C.V.) para os parâmetros analisados da variedade sintética na geração Sint 3 .

\begin{tabular}{lcc}
\hline Variáveis & $\mathbf{F}$ & $\mathbf{C . V . ( \% )}$ \\
\hline Altura de Planta & $2,9068^{*}$ & 13,70 \\
Altura de inserção de espiga & $10,4524^{* *}$ & 14,36 \\
Comprimento de espiga & $2,0335^{\text {ns }}$ & 19,07 \\
Número de fileiras de grãos & $0,7351^{\text {ns }}$ & 14,60 \\
Peso de espiga & $3,8193^{*}$ & 33,55 \\
Peso total dos grãos & $2,7000^{\text {ns }}$ & 35,88 \\
Peso de grãos retidos na peneira $\geq 22$ & $2,1347^{\text {ns }}$ & 81,44 \\
Peso de grãos retido na peneira 20 & $0,5713^{\text {ns }}$ & 69,34 \\
Peso de grãos retidos na peneira <20 & $2,2379^{\text {ns }}$ & 109,84 \\
\hline
\end{tabular}

** Significativo ao nível de $1 \%$ de probabilidade

* Significativo ao nível de $5 \%$ de probabilidade ns não significativo

A Tabela 02, referente aos resultados obtidos com o teste de Tukey, mostra que a variedade em formação não apresentou resultados significativos demonstrando que a mesma já apresenta significativa homogeneidade em relação a altura de plantas, o mesmo pode ser confirmado através do baixo coeficiente de variação.

Tabela 02. Altura de plantas em centímetros $(\mathrm{cm})$ em diferentes blocos da variedade sintética na geração Sint 3.

\begin{tabular}{ll}
\hline Blocos & Altura $(\mathrm{cm})$ \\
\hline 1 & $201.52630 \mathrm{a}$ \\
2 & $226.21050 \mathrm{a}$ \\
3 & $212.52630 \mathrm{a}$ \\
4 & $224.78950 \mathrm{a}$ \\
\hline$M G=216.26316$ & \\
Dms $=25.30455$ &
\end{tabular}

Os dados submetidos ao teste de Tukey (Tabela 03), apresentaram a dispersão em duas classes nítidas, sendo o maior resultado encontrado na parcela 4 , a qual se 
diferencia das outras médias. Apesar do coeficiente de variação demonstrar que essa diferença é baixa, ou seja, as plantas estão apresentando características muito próximas para esse parâmetro.

Tabela 03. Altura de inserção de espiga em centímetros $(\mathrm{cm})$ em diferentes blocos da variedade sintética na geração Sint 3.

\begin{tabular}{ll}
\hline Blocos & Altura $(\mathrm{cm})$ \\
\hline 1 & $114,57890 \mathrm{~b}$ \\
2 & $120,42110 \mathrm{~b}$ \\
3 & $111,36840 \mathrm{~b}$ \\
4 & $140,26320 \mathrm{a}$ \\
\hline $\mathrm{MG}=121,65789$ & \\
Dms $=14,92235$ &
\end{tabular}

Em relação ao parâmetro que a variedade sintética conseguiu obter já 'comprimento de espiga' (Tabela 04), é na sua segunda geração de plantio por não possível confirmar a clara homogeneidade apresentar diferença entre suas médias.

Tabela 04. Comprimento de espiga em centímetros $(\mathrm{cm})$ em diferentes blocos da variedade sintética na geração Sint 3.

\begin{tabular}{ll}
\hline Blocos & Altura $(\mathrm{cm})$ \\
\hline 1 & $14,81579 \mathrm{a}$ \\
2 & $15,78947 \mathrm{a}$ \\
3 & $15,47368 \mathrm{a}$ \\
4 & $17,15789 \mathrm{a}$ \\
\hline
\end{tabular}

$M G=15,80921$

Dms $=2,57545$

Na análise referente ao número de fileiras de grãos nas espigas (Tabela 05), é possível afirmar a clara homogeneidade que a variedade conseguiu obter na sua segunda geração de seleção, os dois resultados mais comuns, com espigas exibindo 14 e 16 fileiras de grãos, respectivamente. 
Tabela 05. Número de fileiras de grãos nas espigas (unid) em diferentes blocos da variedade sintética na geração Sint 3.

\begin{tabular}{ll}
\hline Blocos & Unid \\
\hline 1 & $15.00000 \mathrm{a}$ \\
2 & $15,10526 \mathrm{a}$ \\
3 & $15,63158 \mathrm{a}$ \\
4 & $14,57895 \mathrm{a}$ \\
\hline $\mathrm{MG}=15,07895$ & \\
Dms $=1,88020$ &
\end{tabular}

A análise de variância para o duas parcelas e uma com media mais baixo. parâmetro de avaliação 'peso de espigas' Estas observações são indicadoras da (Tabela 06), apresentou diferenças existência de influência de fatores estatísticas significativas, mostrando que ambientais, e a possibilidade deste uma das parcelas apresenta resultado parâmetro ser passível de melhoria nas superior com posterior resultado mediano de gerações futuras.

Tabela 06. Peso de espigas (g) em diferentes blocos da variedade sintética na geração Sint 3.

\begin{tabular}{ll}
\hline Blocos & Peso $(\mathrm{g})$ \\
\hline 1 & $168,75110 \mathrm{~b}$ \\
2 & $204,73320 \mathrm{ab}$ \\
3 & $186,20370 \mathrm{ab}$ \\
4 & $238,95210 \mathrm{a}$ \\
\hline
\end{tabular}

$M G=199,66000$

Dms $=57,21989$

O resultado obtido através do teste (Sint-2), uma vez que ainda apresenta as de Tukey (Tabela 7) para o parâmetro 'peso diferentes parcelas ainda exibem total dos grãos' demonstra que há uma discrepância entre os fenótipos das plantas, e diferença significativa entre as parcelas o fato desta característica sofrer um amostradas. Esta relativa heterogeneidade pronunciado efeito do meio ambiente. pode ser esperada nessa geração de plantio 
Tabela 07. Peso total dos grãos (g) em diferentes blocos da variedade sintética na geração Sint 3.

\begin{tabular}{ll}
\hline Blocos & Peso $(\mathrm{g})$ \\
\hline 1 & $131,34330 \mathrm{~b}$ \\
2 & $157,33000 \mathrm{ab}$ \\
3 & $149,84790 \mathrm{ab}$ \\
4 & $182,00680 \mathrm{a}$ \\
\hline
\end{tabular}

$M G=155,13200$

Dms $=47,54696$

Para o parâmetro produção de grãos retidos na peneira de malha 22 , o teste Tukey (Tabela 08) não foram encontradas diferenças significativas entre as médias das parcelas, apesar de revelar um coeficiente de variação considerado alto. Esses dados mostram demonstram que a variável ainda poderá ser melhorada em próximas gerações, de modo a se aproximar ainda mais da estabilidade desejada.

Tabela 08. Peso de grãos retidos na peneira de malha 22 (g) em diferentes blocos da variedade sintética na geração Sint 3.

\begin{tabular}{ll}
\hline Blocos & Peso $(\mathrm{g})$ \\
\hline 1 & $131,34330 \mathrm{~b}$ \\
2 & $157,33000 \mathrm{ab}$ \\
3 & $149,84790 \mathrm{ab}$ \\
4 & $182,00680 \mathrm{a}$ \\
\hline $\mathrm{MG}=155,13200$ & \\
Dms $=47,54696$ &
\end{tabular}

A variável peso de grãos retidos por peneira da malha 20 apresentou homogeneidade no teste Tukey entre os tratamentos (Tabela 09), igual resultado ao encontrado para a retenção de sementes pela malha 22 .

Tabela 09. Peso total dos grãos retidos na peneira de malha 20 (g) em diferentes blocos da variedade sintética na geração Sint 3.

\begin{tabular}{ll}
\hline Blocos & Peso $(\mathrm{g})$ \\
\hline 1 & $67,77737 \mathrm{a}$ \\
2 & $74,31210 \mathrm{a}$ \\
3 & $68,47053 \mathrm{a}$ \\
4 & $114,10950 \mathrm{a}$ \\
\hline
\end{tabular}

$M G=81,16737$

Dms $=56,46235$ 
Na última sub variável dentro do peso de grãos de malhas inferiores a 20 compartilhou dos mesmos resultados encontrados para as outras dimensões de peneiras, não apresentando diferenças significativas teste Tukey (Tabela 10), reforçando assim os comentários e discussões que foram descritos anteriormente nesse trabalho.

Tabela 10. Peso total de grãos retidos na peneira de malha inferior a 20 (g) em diferentes blocos da variedade sintética na geração Sint 3 .

\begin{tabular}{ll}
\hline Blocos & Peso $(\mathrm{g})$ \\
\hline 1 & $41,98737 \mathrm{a}$ \\
2 & $45,13421 \mathrm{a}$ \\
3 & $46,92684 \mathrm{a}$ \\
4 & $55,30947 \mathrm{a}$ \\
\hline $\mathrm{MG}=47,33947$ & \\
Dms $=28,03956$ &
\end{tabular}

\section{DISCUSSÃO}

As populações de milho com potencial para serem utilizadas como genitoras em programas de melhoramento intrapopulacionais devem possuir altas médias e ampla variabilidade genética quanto as características de interesse (HALLAUER; MIRANDA FILHO, 1988). Assim, podemos afirmar que os resultados obtidos nas variáveis estudadas estão plenamente satisfatórios e podem ser considerados como plenamente esperados para um programa de melhoramento que ainda se encontra na segunda geração de intercruzamento aleatório e recombinação genética. Estes resultados demonstram os ganhos relevantes já conquistados em relação às características dominantes selecionadas previamente em condições de campo, tanto no que diz respeito ao desenvolvimento da mesma e também nas gerações passadas.

Pacheco et al. (1998), trabalhando com diversas populações sintéticas, encontraram valores de altura de plantas variando de $1,50 \mathrm{~m}$ a 2,50 $\mathrm{m}$ e altura de inserção de espiga variando de $0,8 \mathrm{~m}$ a 1,50 m. Com isto, podemos verificar que a segunda geração da variedade em estudo apresenta altura de planta e altura de espiga com valores satisfatórios, além de uma relativa homogeneidade das mesmas entre plantas e parcelas, deixando claro que, conforme as próximas gerações de estudo forem produzidas, a tendência das plantas será de se tornarem valores cada vez mais estáveis para essas variáveis morfológicas. 
Segundo Mumm et al. (1994), as diferentes técnicas de classificação dos mesmos genótipos, sejam com informações genealógicas, moleculares ou agronômicas, devem apresentar resultados similares se as classificações refletirem a verdadeira associação entre os valores genótipos. Este fenômeno possibilitaria, assim, garantir através de valores igualitários apresentados nas análises estatísticas, a evolução do genótipo da planta. Além disto, estaria correlacionado com seu desenvolvimento genético e possibilita a variedade obter as melhores características de cada uma das variedades utilizadas para a população inicial, e que foram selecionadas através de avaliação a campo.

No caso das variáveis que apresentaram ainda algumas diferenças significativas entre as parcelas, pode-se demonstrar de forma clara a importância dos estudos publicados por Hallauer e Miranda Filho (1988), uma vez que deve-se preferir cruzamentos entre populações ou genitores que apresentam altas médias e divergência ampla nas características de interesse, deixando claro que conforme a conclusão de cada geração de seleção, a variedade sintética resultante acaba por se distanciar de cada um dos fatores característicos das cultivares iniciais, formando a sua própria identidade agronômica.

Em relação aos coeficientes de variação apresentados, segundo Scapim et al. (1995), os coeficientes de variação que oscilaram de $7,0 \%$ a $20,3 \%$, conferem uma boa precisão aos experimentos, demonstrando que, em sua maioria, os resultados das análises foram satisfatórios e apresentaram valores aceitáveis para embasar fortes argumentos que levam a acreditar na superioridade e adaptação da variedade em estudo (Figura 13).

Como descrito por Ribeiro et al. (2000), resultados de inúmeros trabalhos com a cultura do milho permitem inferir não haver relação fixa entre a homogeneidade ou heterogeneidade de determinado genótipo e sua estabilidade, sendo possível selecionar cultivares mais estáveis em qualquer grupo, quer sejam variedades, híbridos simples, hibrido triplo ou hibrido duplo. 


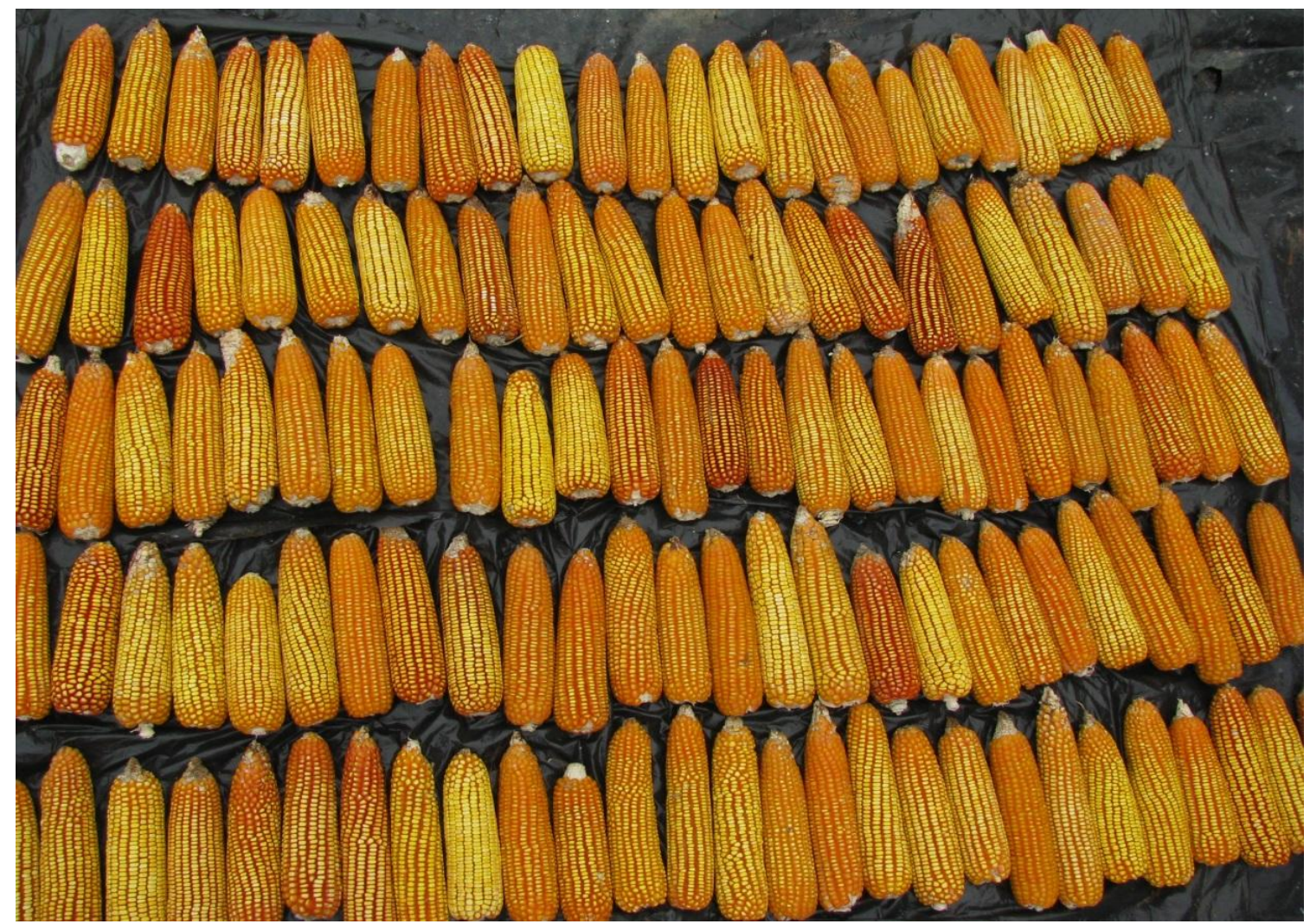

Figura 1. Espigas colhidas no segundo ciclo de seleção e que formarão a população Sint-3, da variedade sintética de milho descrita no presente trabalho.

Foto: $\mathrm{O}$ autor.

\section{CONCLUSÃO}

O presente trabalho evidenciou $\mathrm{o}$ avanço na homogeneidade genética da variedade de milho que está sendo desenvolvida, comprovando a elevação no grau de estabilidade de diversos parâmetros agronômicos após sucessivos ciclos de seleção. Para todas as características fenotípicas avaliadas, pôde-se comprovar uma contínua uniformidade e elevação na qualidade fenotípica já na geração Sint 3 da variedade, estimulando a continuidade do programa de melhoramento e objetivando a obtenção de uma variedade comercial.

\section{REFERÊNCIAS}

BARBOSA , J.A. Influencia de espaçamento e arquitetura foliar no rendimento de grãos e outras características agronômicas do milho (Zea mays L.). 1995. 48f. Dissertação (Mestrado em Agronomia, Fitotecnia) Universidade Federal de Lavras, Lavras.

DOURADO NETO, D.; FANCELLI, A. L. Produção de milho. Guaiba: Agropecuária, 2000.

HALLAUER, A.R.; MIRANDA FILHO, J.B. Quantitative genetics in maize breeding. Ames: lowa State University, 1988. 468p.

HORN, D. Cinética da absorção de nutrientes em cultivares de milho com diferentes bases genéticas e seus desempenhos agronômico e econômico em quatro níveis de manejo. 2004. 111f. Dissertação (Mestrado em Ciência do Solo) - Curso de Pós-graduação 
em Ciência do Solo, Universidade do Estado de Santa Catarina.

MALACRIDA, L.L.G.; ROMEU, A.S.; LEBEDENCO, A.; MARQUES, T. A.; TIRITAN, C.S. Avaliação regional de 16 cultivares de milho (Zea mays L.) no período de 30 de novembro de 2001 a 10 de março de 2002. In: ENCONTRO ANUAL DE PESQUISA INSTITUCIONAL NA UNOESTE, VII., 2002, Presidente Prudente. Anais... Presidente Prudente: Pró-reitoria de pesquisa e pósgraduação da Universidade do Oeste Paulista, 2002. p.1-11.

MUMM, R.H.; HUBERT, L.J.; DUDLEY, J.W. A classification of $148 \mathrm{U}$. S. maize inbreds - II: validation of cluster analysis based on RFLPs. Crop Science, Madison, v.34, p.852-865, 1994.

http://dx.doi.org/10.2135/cropsci1994.0011 $\underline{183 \times 003400040006 x}$

MUNDSTOCK,C.M.; SILVA, P.R.F. Manejo da cultura do milho para altos rendimentos de grãos. Porto Alegre: Universidade Federal do Rio Grande do Sul, 2005. 50p.

PACHECO, C.A.P.; GAMA, E.P.; GUIMARÃES, P.E.; SANTOS, M.X.; FERREIRA, A.S. Estimativas de parâmetros genéticos nas populações CMS-42 e CMS-43 de milho pipoca. Pesquisa Agropecuária Brasileira, Brasília, v.33, n.12, p.1995-2001, dez. 1998.

PATERNIANI, E. Novos desenvolvimentos no melhoramento vegetal. In: OSUNA, J. A.; MORO, J. R. Produção e melhoramento do milho. Jaboticabal: FUNEP, 1995 . Cap. 8, p.117-126.

RIBEIRO, P.H.E.; RAMALHO, M.A.P.; FERREIRA, D.F. Adaptabilidade e estabilidade de cultivares de milho avaliadas em diferentes condições ambientais. Pesquisa Agropecuária Brasileira, Brasília, v.35, n.11, p.2213- 2222, nov. 2000. http://dx.doi.org/10.1590/S0100204X2000001100013
SCAPIM, C.A.; CARVALHO, C.G.P.; CRUZ, C.D. Uma proposta de classificação dos coeficientes de variação para a cultura do milho. Pesquisa Agropecuária Brasileira, Brasília, v.30, n.5, p.683-686, maio 1995.

SILVA, F. de A. S. e. The ASSISTAT Software: statistical assistance. In: INTERNATIONAL CONFERENCE ON COMPUTERS IN AGRICULTURE, 6, Cancun, 1996. Anais... Cancun: American Society of Agricultural Engineers, 1996. p.294-298.

SILVA, F. de A. S. e. \& AZEVEDO, C. A. V. de. Versão do programa computacional Assistat para o sistema operacional Windows. Revista Brasileira de Produtos Agroindustriais, Campina Grande, v.4,n.1, p71-78, 2002.

SILVA, F. de A. S. e. \& AZEVEDO, C. A. V. de. A New Version of The Assistat-Statistical Assistance Software. In: WORLD CONGRESS ON COMPUTERS IN AGRICULTURE, 4, Orlando-FL-USA: Anais... Orlando: American Society of Agricultural and Biological Engineers, 2006. p.393-396.

SILVA, F. de A. S. e. \& AZEVEDO, C. A. V. de. Principal Components Analysis in the Software Assistat-Statistical Attendance. In: WORLD CONGRESS ON COMPUTERS IN AGRICULTURE, 7, Reno-NV-USA: American Society of Agricultural and Biological Engineers, 2009. 